\title{
Adipose-derived stem cells for cartilage regeneration - moving towards clinical applicability
}

Luminita Labusca ${ }^{1,2^{*}}$ and Kaveh Mashayekhi $i^{1,2,3}$

See related research by Van Pham et al., http://stemcellres.com/content/4/4/91

\begin{abstract}
Despite multiple methods of treatment and a wealth of research in the field of regenerative medicine focusing on cartilage defects, the management of cartilage injuries remains a challenge. A recent study by Van Pham and colleagues proposes a method for preconditioning autologous adipose-derived stem cells. Their study offers evidence about the increased proliferative and chondrogenetic capabilities of platelet-rich plasma-treated adipose-derived stem cells and the increased efficiency of these in treating articular cartilage defects in mice. Even though the method needs further elaboration and the composition of the repair tissue requires investigation, the results are promising for the design of clinically acceptable cell therapies aimed at cartilage regeneration.
\end{abstract}

\section{Commentary}

Rome wasn't built in a day

Articular cartilage injuries represent an increasing threat to the physical, professional and social performance of individuals and a burden for healthcare resources. Cartilage lesions of various degrees of severity can be simple or associated with multiple articular, periarticular or systemic, post-traumatic or degenerative conditions. Regardless of their diversity, several common problems need to be considered: the provision of immediate symptomatic improvement, the evaluation of rehabilitation time and the prediction of medium- and long-term outcome in the affected joint, in the context of patient-specific demands

\footnotetext{
* Correspondence: drlluminita@yahoo.com

${ }^{1}$ Department of Orthopedic Surgery, University Hospital Saint Spiridon, lasi 700111, Romania

${ }^{2}$ SBIM Systems Bioinformatics and Modeling, GmbH, Basalt str. 39, D-60487, Frankfurt, Germany

Full list of author information is available at the end of the article
}

for functional recovery. Current therapies provide good short-term improvement within acceptable time frames; however, medium- and long-term improvement in the injured cartilage is still hampered by the occurrence of post-traumatic arthritis.

Different tissue engineering or cell therapy scenarios have been proposed, raising hope for possible complete structural and functional joint resurfacing in the near future. Bringing such expectancy closer to clinical applicability, a recent study by Van Pham and colleagues [1] reports a method of using adipose-derived stem cells (ADSCs) preconditioned with activated platelet-rich plasma (PRP) as combined cell therapy for the management of post-traumatic articular cartilage defects. Due to the ubiquity of the tissue of origin and their ease of procurement and proliferative capability, ADSCs are regarded as a convenient cell source for regenerative medicine [2]. The use of ADSCs for cartilage regeneration, however, appears to be problematic. ADSC chondrogenetic potential seems to be lower than that of bone marrow-derived mesenchymal stem cells [3]. ADSCs, moreover, can inhibit cartilage regeneration in vivo via vascular endothelial growth factor (VEGF)-A-mediated chondrocyte apoptosis and reduced proteoglycan synthesis [4].

The study by Van Pham and colleagues [1] proposes that ADSC chondrogenic potential can be enhanced and VEGF release can be reduced by supplementing the culture media with PRP. Being rich in growth factors, autologus PRP is currently used as a 'biological' augmentation in various clinical applications, including cartilage repair [5]. Used as a culture media supplement, buffered PRP has been shown to enhance human mesenchymal stem cell proliferation and chondrogenic differentiation in vitro (leading to ten-fold increased Sox-9 and aggrecan mRNA expression and a two-fold increase in RUNX2 mRNA) [6]. In another study, a rapidly degrading PRPderived scaffold seeded with autologus ADSCs or bone marrow-derived mesenchymal stem cells was used to 
treat osteochondral lesions in a rabbit model [7]. A significant improvement in macroscopic and histological International Cartilage Repair Society score was reported, as well as the presence of type II collagen-expressing, enhanced green fluorescent protein-labeled ADSCs within the defect at 9 weeks after implantation [7]. In the study by Van Pham and colleagues, human ADSCs preconditioned with PRP were used as combined cell therapy, together with autologus PRP, for the treatment of fresh articular cartilage defects in NOD/SCID mice. ADSCs were apparently proven to display enhanced proliferative capability in vitro when cultured with an optimal concentration of $15 \%$ autologus calcium-activated PRP replacing fetal bovine serum in the culture media. Chondrogenesis-related gene expression (collagen type II, Sox-9 and aggrecan) increased in the PRP- compared to fetal bovine serum-treated cells and VEGF concentration in the conditioned media was significantly reduced.

These results are promising, as they offer evidence for a practical modality of obtaining autologous stem cells for clinical application. The method requires relatively few cell manipulations; it does not need media supplements of animal origin but does involve a waiting time for cell expansion and implies the need for a good manufacturing practice facility. However, the precise composition of PRP has to be further detailed (degrees of platelet enrichment compared to peripheral blood, presence or absence of white blood cells) as these parameters are dependent on the PRP extraction method. Growth factor concentration is dependent on the degree of platelet enrichment while white blood cells can exert catabolic effects on articular cartilage [5]. The gene expression profile of PRP-treated ADSCs also has to be further investigated, to clarify the situation of osteogenesis-related genes, and to eliminate the possible induction of a sub-population of osteoblast precursors.

ADSCs preconditioned with PRP in the study of Van Pham and colleagues proved to be efficient in treating cartilage defects in mice. Functional recovery time and the area of injured cartilage were significantly decreased, while the number of chondrocyte layers increased 45 days after cell administration. It is possible the injected cells could have had a structural role in cartilage repair as previously reported [7], contributing to filling the defects and inhibiting angiogenesis in vivo [8]. Further studies will be needed to ascertain the role of ADSCs in chondrogenesis and to investigate the composition and properties of the regenerated cartilage (such as collagen fiber type, distribution, and mechanical properties). This is crucial for clinical applicability as it would enable estimation of the recovery time and improve medium- and long-term outcomes.

Despite the rather large number of choices for treating cartilage defects, current therapies provide for, at most, fibrous cartilage repair or tissue prone to endochondral ossification [9]. Such repairs have low biomechanical properties and are prone to failure in the long run, preventing complete functional recovery and professional rehabilitation. For reasons like reduced contamination and decreased intervention time, the infusion of a cell suspension could be more feasible than implanting a cell-seeded scaffold. Used as a method for cartilage defect treatment and arthritis prevention [10], cell therapy has the potential of attracting clinical acceptance. Proof of a cell population homing within the defect and participating in de novo hyaline cartilage formation would eliminate the need for scaffold implantation, thus overcoming issues related to graft integration, peeling, and/or ossification.

\section{Abbreviations}

ADSC: Adipose-derived stem cell; PRP: Platelet-rich plasma; VEGF: Vascular endothelial growth factor.

\section{Competing interests}

The authors declare that they have no competing interests.

\section{Author details}

${ }^{1}$ Department of Orthopedic Surgery, University Hospital Saint Spiridon, lasi 700111, Romania. ${ }^{2}$ SBIM Systems Bioinformatics and Modeling, GmbH, Basalt str. 39, D-60487, Frankfurt, Germany. ${ }^{3}$ BioTalentum, Aulich Lajos str. 26, 2100, Gödöllö, Hungary.

\section{Published: 1 October 2013}

\section{References}

1. Van Pham P, Bui KH, Ngo DQ, Vu NB, Truong NH, Phan NL, Le DM, Duong TD, Nguyen TD, Le VT, Phan NK: Activated platelet-rich plasma improves adipose-derived stem cell transplantation efficiency in injured articular cartilage. Stem Cell Res Ther 2013, 4:91.

2. Mizuno $H$, Tobita $M$, Uysal AC: Concise review: Adipose-derived stem cells as a novel tool for future regenerative medicine. Stem Cells 2012, 30:804-810.

3. Boeuf S, Richter W: Chondrogenesis of mesenchymal stem cells: role of tissue source and inducing factors. Stem Cell Res Ther 2010, 1:31.

4. Lee CS, Burnsed OA, Raghuram V, Kalisvaart J, Boyan BD, Schwartz Z: Adipose stem cells can secrete angiogenic factors that inhibit hyaline cartilage regeneration. Stem Cell Res Ther 2012, 3:35.

5. Kon E, Filardo G, Di Matteo B, Marcacci M: PRP for the treatment of cartilage pathology. Open Orthop J 2013, 7:120-128.

6. Mishra A, Tummala P, King A, Lee B, Kraus M, Tse V, Jacobs CR: Buffered platelet-rich plasma enhances mesenchymal stem cell proliferation and chondrogenic differentiation. Tissue Eng Part C Methods 2009, 15:431-435.

7. Xie X, Wang Y, Zhao C, Guo S, Liu S, Jia W, Tuan RS, Zhang C: Comparative evaluation of MSCs from bone marrow and adipose tissue seeded in PRP-derived scaffold for cartilage regeneration. Biomaterials 2012, 33:7008-7018.

8. Bara JJ, McCarthy HE, Humphrey E, Johnson WE, Roberts S: Bone marrowderived mesenchymal stem cells become anti-angiogenic when chondrogenically or osteogenically differentiated: implications for bone and cartilage tissue engineering. Tissue Eng Part A 2013 [Epub ahead of print]. PMID 23895198.

9. Gelse K, Beyer C, Welsch Gand Blanke M: Endochondral ossification in cartilage repair tissue hampers bone marrow stimulating techniques. Rheumatol Curr Res 2012, S3:002.

10. Tuan RS, Chen AF, Klatt BA: Cartilage regeneration. J Am Acad Orthop Surg 2013, 21:303-311.

\section{doi:10.1186/scrt329}

Cite this article as: Labusca and Mashayekhi: Adipose-derived stem cells for cartilage regeneration - moving towards clinical applicability. Stem Cell Research \& Therapy 2013 4:118. 